\title{
Artificial Intelligence Waves on Space Computation Management: A Review Report
}

\author{
Mostafijur Rahman ${ }^{1}$, Ruhul Amin ${ }^{2 *}$ \\ ${ }^{1}$ Lecturer, Department of Business Administration, First Capital University of Bangladesh, Chuadanga, BANGLADESH \\ ${ }^{2}$ Senior Data Entry Control Operator (IT), ED-Maintenance Office, Bangladesh Bank (Head Office), Dhaka, BANGLADESH \\ *Corresponding Contact: \\ Email: ruhulaminshuzon@gmail.com
}

\begin{abstract}
When writing about the history of the internet, it is important to note that aerospace was among the significant pioneers in computer networking computer network for private was used in first airline reservation system "SABRE" in 1960 for American airlines. While sage was the first computer system in the world, its deficiencies led to the development of ARPANET. These systems formed the foundations for the internet and the development of other computer programs in aerospace, any deficiency led to the invention of a new program, giving birth to programming, $\mathrm{CAD}$, and CAM that brought about simulations. Aerospace computing has evolved over the years and is now carrying the whole weight of the aerospace industry. Before the launch of any space vehicle or satellite, simulation has become a necessary step, checking for weaknesses for corrections to be done on the ground. Besides, computer simulation has been essential in training, facilitating the training of pilots worldwide. This article presents more information regarding the application of AI in aerospace computing, flight simulations, and their advantages in the aerospace industry.
\end{abstract}

Key words:

Artificial Intelligence, Waves Management, Space Computation

\section{INTRODUCTION}

Complex design problems in engineering are multi parameter optimizations, which require the use of physics models to predict outcomes of several input parameters. However, design depends desiring a particular outcome and generating suitable parameters to achieve them. While it is not feasible to invert science models to achieve optimal results, favorable combinations of inputs can be extracted by parallelizing the problem into a large population of parameters and then compete the results. The advantage behind this approach is that engineers and other experts in different fields can design complex systems and adapt them 
to their natural environment (through simulation) just like evolving and living organisms adapt to their environments. Over the years, computationally derived evolutionary designs have had significant competitiveness with human created designs in creativity, robustness, and complexity. This has been shown through low thrust trajectory optimization, power systems design, robotic arm deployment path finding, mission planning and scheduling, avionics architecture design, and neural network design, among others.

In 1957, the launch of two Sputnik satellites by the Soviet Union came as a surprise to most Americans. While the intelligence community did not take it as a surprise, most ordinary Americans did, with the two satellites demonstrating that the Soviet Union did not only lead the United States (US) with booster rockets, but also powerful satellites (O'Regan, 2016). The launching attracted many responses, including the founding of the agencies such as the US Defense department and a civilian agency. The Advanced Research Projects Agency (ARPA), currently known as DARPA was among the significant responses, which was meant to ensure the US does not face similar issues like after the launch of the two satellites. ARPA did this by creating a large platform for long term research among other functions. While functions like space and missile exploration were assigned to ARPA, those duties were transferred to the National Air Space Administration (NASA) in 1958. Since this time, one can count remarkable achievements and advancements in space exploration, with many countries following the lead to launch satellites and other space systems.

In the beginning of the 1960s, DARPA designed and created a transforming system, APRANET (a network of computers), which formed the foundation for today's internet. By responding to challenge offered by President John Kennedy, NASA landed several astronauts to the moon and back safely (O'Regan, 2016). The most transformations in the use of internet occurred in 1990s when the internet moved from network known by scientists and other experts to a network that could be used by any ordinary citizen to perform several operations. In about half a century since NASA and DAPRA, one can revisit any achievements in space exploration and computing, giving relationships of both technologies in achievements we enjoy today. There has been tremendous progress in both computing and aerospace, but clearly, the future did not turn out as the scientists expected. In 1960s, some scientist realized and made predictions that computers would one day attain artificial intelligence, becoming human servants or companions. Fiction writers embraced the idea and portrayed such computers as our beneficial servants, for example robots in movies, or as detriment as in malevolent computer in the movie 2001(O'Regan, 2016). While such artificial intelligence has not been achieved fully, no one appears to be surprised by it. The internet, wireless telephones, personal computers, among other advances has brought computing technology that surpasses the imagination of scientists and experts in the past. Although humans cannot communicate with such devices like with other humans, they have a surprising intelligence, facilitated by high processing power and memory than even human brain.

There are numerous predictions that were made in the past regarding computer artificial intelligence and space exploration that have not yet come to pass. Apollo missions to the moon resulted in predictions such as permanent outposts in the moon, managed mission to mars, and tourist hotels in earth's orbit. Currently, none of the predictions have come to pass (Donepudi, 2018). However, advances in space technology have evolved tremendously. The earth has numerous weather satellites that are significant parts of human daily lives. In the realm of space exploration, the Global Positioning system (GPS), has been developed to offer precise positioning and timing location services in short time and low costs (O'Regan, 
2016). Additionally, there have been space robotic explorations in mars and other planets that are more comprehensive than in the past. In the realm of computing, advances in processing power and sheer memory capacity have been done together with networking. Jointly, they have covered most frustrations regarding failure of developing computer with intelligence as human beings. This is unlike the realm of space exploration, where frustration is still an issue even with advancements discussed above. There have been problems with aircrafts, with their makers working hard to avoid accidents and complications when ferrying people or products.

\section{CAD AND CAM}

In the past sixty years, aerospace and computing have had close relationship, and hence hard to treat their history separately. The invention of digital computers in the period between 1940- 1950 was connected to solution of most problems in aerodynamics and astronomy. Also, they served to support technologies of aircraft design and production, air anti weapons, air traffic control, and missile development guidance. The aerospace community commanded large sums of money from the US government after 1945, which pushed the development of digital computing in fast way. In general terms, a digital computer is a multipurpose device that can do many operations when fed with suitable program. After their development, digital computers experienced tremendous developments in power and memory, and found their applications in aerospace. In 1950s, these computers were coupled with general purpose software such as programming language FORTRAN, which was used for computer aided design and computer aided manufacturing (CAD and CAM) (Linger, 2018). Unlike in other field of application such as banking and finance, aerospace applications have additional constraint. Up to 1960, computers were fragile, large in size, and consumed tremendous amount of power. While they were still applicable, the US took the responsibility of making computers universal through heavy capital investments. They aimed at addressing issues like size, readability, ruggedness, weight, among others.

Air force and NASA have had significant direct influence on computing, including the development of CAD. With the help of funding form the Air force at Massachusetts Institute of Technology (MIT), machine tools were controlled by sequence of digital computer controls. The results of this were transformation in machine tool working, not only in aerospace but also in metal working in general. Working in different working stations, different NASA engineers had been using digital computers for stress analysis of aircraft and rockets. Vehicles had to be strong enough before launching to hold oxygen and fuel, and support the structure of the upper stages, while withstanding the vibrations and stress during launching (O'Regan, 2016). As a result, they had to be light in weight. Before then, aircraft engineers had been struggling with stress testing problems for many decades. For a typical aircraft company, stress testing had to go through ten engineers for analysis and confirmations. The reason for such many engineers was to ensure the aircraft was strong enough to withstand its weight and contents and light enough to take off the ground. NASA took advantage of this and funded the development of digital computers in this field. A stress analysis program was developed (NASTRAN) which was based on FORTRAN, and to date, it has become a standard to do stress analysis through such programs in aerospace industry. 


\section{Typical journey to space}

Control is a significant component Space craft and guided missiles propulsion. While missiles are launched and controlled like aircrafts, they do not need a human pilot. Guided missiles or aircrafts engines have to operate continuously, while working against atmospheric drag, with the forward motion of the wings through air generating lift to counter gravitational force (Lee et al., 2017). Unlike these two systems, a rocket counters the gravitational force by detect application of thrust. However, once a spacecraft enters the space, there is no atmospheric drag and hence the engines shut off. As a result, the engines work for a little fraction of mission time. However, control is required but in a different way, depending on the phase of the mission. During the powered phase of the mission, which may last for few minutes, the challenge is always aligning the thrust vector with the center of gravity of the vehicle (for a rocket) (Sucharov, 2018). In most cases, the configuration of the rocket is unstable, with the engines at the bottom and the payload and fuel tanks above. Due to this configuration, such a rocket will topple instantly unless constantly and actively guided as it ascents. Once the first order stability is achieved, the system of the vehicle may direct the thrust to deviate from alignment as the rocket gains velocity. In doing so, the vehicle tilt to an optimum angle where it does not only counter gravity but also propels horizontally to achieve orbit, to escape the earth entirely, or to return to earth with some distance.

One a spacecraft reaches the required velocity; it costs to its destination through ballistic trajectory, as the path resembles that of a thrown rock. The assumption of this is that the desired velocity was right at the end of powered phase (when engines are cut off). If this does not happen, main or auxiliary engines are powered to change the vehicle's trajectory. During this period, the space craft is fired occasionally rather than continuously. This is because atmospheric drag is absent, requiring little force to change trajectory (Lee et al., 2017). The process may be continuous, involving determining whether the vehicle is in the right path and acting accordingly. Once the motors of the spacecraft are powered off, it can go in any direction no matter how it is pointed. However, a practice mission requires that any vehicle orients in a specific way such as pointing a camera to a spot on earth, point solar panels to the sun, and so on (Sucharov, 2018). As explained above, space craft will achieve control through rocket motors with very insignificant thrust such as activating an instrument, turning on a camera, or preparing a vehicle for capture by another planet. This can be done automatically by people onboard or from control stations on the ground. The flight by Wright brothers was unstable in design, and hence required continuous attention from the pilot.

Navigation can often be done at slower pace, allowing careful process of radar data by powerful mainframe computers and then radiod up accordingly (Sucharov, 2018). While the main control is done by onboard gyroscopes and accelerometers with no communication form any direction, control and navigation makes use of signals from and to the ground stations as well. The translation of the signals from an integrating accelerometer or gyro is called computing. Most of these operations require extensive and complex calculations which can only be achieved through computing. For example, the aligning of the space craft in the first phase requires calculation to be done at a fast rate, almost similar to the action of the rocket. Making a mistake at this stage may bring severe consequences to the people onboard as well as the while mission. Computing provides the required infrastructure and computing power through artificial intelligence for easy control of space vehicles during launching and during the entire mission. With simulation using CAD, aerospace engineers 
are able to determine the correct design parameters to use and adapt their systems to the expected conditions in the space. As a result, even before a vehicle is launched into the space, they are sure it will adapt to the natural conditions of the space region.

\section{Evolutionally computation framework and an example of simulation}

To evaluate the fitness of a particular design and implement the evolutionary cycle, the JPL power analysis tool (MMPAT) within the PERSON evolutionary computing framework is used. The Multi Mission Power Analysis Tool (MMPAT) is a tool responsible for the modeling of the behavior of behavior of power sources in space craft and energy storage devices within the vehicle as they interact with environment and spacecraft loads throughout the mission timeline (Gorur \& Calli, 2018). Different possible parameters are fed to the design of the system, noting down the changes and possible cases of failure after launching. The developers are therefore able to determine points of weakness and make corrections accordingly, either in the design or choose fitting parameters to the existing environment. The decision depends on the easy way of solving the problem. This approach is currently used in Mars Exploration Rover (MER) operations in the prediction of power subsystem resources before uploading sequence of activities. Through this tool in evolutionary computing framework, it is possible to provide several optimized designs regarding the anticipated subsystem performance instead of using estimates which are associated with errors.

\section{COMPUTER SimULATION}

Computer simulation is a process of mathematical modeling, designed to predict the outcome or behavior of a physical system or a real world. Because they check reliability of mathematical models, they have become very popular tools for mathematical modeling of numerous natural systems in computational physics, climatology, astrophysics, and in other fields. The simulation of a system is presented as the running of the systems model. Simulation can be used in the exploration and in gaining new insights into new technology. Additionally, it can be used to estimate the performance of different systems that are too complex for analytical solutions. These simulations are realized on running computer programs that are either small or large scale. Small programs run on small devices for short time while large scale programs can run for hours or days, depending on the groups of computers. The scale of events possible through computer simulations have so far exceeded human expectations and anything possible using paper and pencil mathematical modeling that was used traditionally (Donepudi, 2016). Simulation technology has come a long way since this traditional ways as they were once science fiction but today they are part of human daily life. Their impact goes beyond technology for the sake of technology. Modeling and simulation techniques play important roles in many industries, especially the aerospace industry.

The aerospace industry has benefited significantly from numerous successful simulations up to date. Currently, simulation hardware and software give tremendous technological benefits to aviation students, experienced experts in the industry, including aerospace manufacturers (Linger, 2018). While flight simulators are not new, anyone can have a computer with different simulation programs such as Microsoft Flight Simulator. However, the evolving technology continues to set new limits of which can be achieved through computer simulation in real life. Most of the leading aerospace training and engineering industries are investing significantly in simulation for its benefits. For example, the US air 
force spent invested over 20 billion dollars and recruited the help of 25 simulation companies countrywide. The aim was to improve their simulation and training acquisition and also the procurement process of the companies. When considering the aspect of the cost saving, simulation is associated with the greatest benefits. Reports and research show that training at a simulator is $20 \%$ less expensive that training physically at the field (Lewis, 2016). Air force pilot training programs worldwide depend on flight simulation, with over one third of all pilots using simulator based training.

\section{Effectiveness OF Flight SimUlators for Training}

Flight simulators are critical components of pilot training programs. In some instances, simulator based training is seem as more effective than training in areal air plane. However, it is important to note that this does not diminish the need to experience training in a real aero plane (Lewis, 2016). It just highlights how much of experience is transferrable. There are two major benefits associated with training in a simulator. Firstly, all training objectives can be achieved using simulator alone. Secondly, the simulator prepares the pilot to the real world aircraft training through insight and familiarity. Both of these reduce the costs of pilot training, increase the effectiveness of modeling, and simulation programs. To show the effectiveness of these technologies, the US Air force's training program is the best example. After making use of these technologies, trainees become qualified in about half the time they used to take in real life training. Students are able to learn at their own pace using simulation based training. They can receive detailed feedbacks from their instructors in every stage (Donepudi, 2019). Also, they have opportunities to rectify their mistakes in safe simulators before they set foot in real crafts. Additionally, the instructors can make use of multitude of scenarios in the preparation of trainees as well as experienced pilots. The costs of losing a trainee or a plane during training are usually high. Simulators can replicate such severe conditions repeatedly while the operators familiarize with them to be able to handle planes in complete safety.

\section{Advantages of Simulation before Launching Space Vehicles, Satellites, AND IN TRAINING}

Compared to simulator based training, overhead training is associated with higher costs. Aircrafts require high amount of fuel and extensive maintenance for safety and to ensure it runs properly. Flight simulators reduce the number of lessons required for a trainee, making programs more accessible and affordable (Lewis, 2016). In the United Kingdom, it is mandatory for all pilots to undergo two days training in a flight simulator. Without the simulators, the constant pilot training would cost airlines and governments millions of dollars. For an airline with 1000 pilots, the cost of training would exceed 60 million dollars annually. In comparison, flight simulators cost less than $10 \%$ of this figure. Without simulators, many airlines globally would struggle to remain successful in the industry.

\section{Environmental concerns}

There are many concerns surrounding our environment and climate change in the whole world regarding the reduction of carbon emissions as much as possible. Most companies dealing with vehicle have to function under the law of conserving environment. This includes producing vehicles without or with insignificant carbon emissions on the environment. The same applies to the aviation and aero planes industry. Globally, aviation 
produces 859 million tons of carbon (IV) oxide. Simulators have played a role of reducing this value recently, and will continue reducing it as simulators and VR technologies advance in the future. A typical flight simulator will run for twenty hours daily. Applying the same workload to an air plane would be hard and the emissions would be tremendous. Simulators therefore reduce environmental pollution and noise pollution.

\section{Safety concerns}

Simulations are the best option when it comes to practicing dangerous situations, For example, when operating an aircraft under specific type of failure or performing an abnormal procedure. For example, a trainee can learn what to do when a helicopter's engine fails during takeoff. Studies have shown that simulation technologies have significantly improved the abilities of pilots to handle unexpected events during practice (Lewis, 2016). Training in a real plane under such conditions is impossible due to safety reasons. The pilot cannot have opportunities to learn from such failures since results are catastrophic. Also, the community is concerned of their safety around the training center. Some training operations mat fail, resulting to death and destruction of property, which is unacceptable. Simulations are suitable as they remain in a constant place, ensuring before a trainee can launch a plane, he/she has already experienced typical conditions on the ground.

Most of the above advantages revolve around training. It is important to note that simulation is applied to other areas as well. With increased artificial intelligence of digital computers, it is now possible to subject different models of planes, satellites, and space crafts to the conditions of the desired destinations (Donepudi et al., 2020). For example, a plane will be simulated to conditions that are found in the atmosphere, a satellite will be simulated to conditions of the space and space ships. The simulation includes the time each vehicle spends on particular conditions, possibly that is different from those of the earth. The aim as discussed earlier is to test if the vehicle will withstand the conditions or not. If not, corrections are done according to remove any chances of failure once the vehicle is launched to the respective destination.

The limitations of human brain and bodies will continue dragging back human efforts to dig deep into space exploration. Even with the many laws of physics we know, it is hard to envision traveling beyond the orbit of the mars. The same applies even with most optimistic extrapolations of chemical rocket propulsion. If current development in computers continues in the same pace, computers will possibly contain similar number of circuits as there are neurons in human brain in the coming years. If this happens, one would envision transferring nature of human consciousness to computers. This argument is supported by different inventors such as ray Kurzweil, who believe this is inevitable. While this is still depends on equivalence, humans have experienced artificial intelligence at higher levels than it was predicted few decades ago. While having more circuits may not be enough to cross intelligence, consciousness will probably be transferred. Much advancement has been achieved in aerospace industry through computations and probably, more will come in few years to come. The development of computers is making most operations easy than never before.

\section{ConCLUSION}

Aerospace computing has taken lead in most aerospace operations. Some of the operations require complex calculations that can only be solved through digital computers. While current technologies in the industry are effective, the future seems to hold tremendous 
advancements, especially with rapidly improving technology. Simulation forms a significant part of aerospace training globally. It has been accepted and utilized in different institutions and business in the aerospace industry. As the simulation capabilities continue to grow, their applications in design, procurement, training, and development will grow likewise. As they become more detailed, advanced, and refined, the effectiveness of the technology to provide realism, skill, and training transfer in real space systems will also increase.

\section{REFERENCES}

Donepudi, P. K. (2016). Influence of Cloud Computing in Business: Are They Robust?. Asian Journal of Applied Science and Engineering, 5(3), 193-196. http://doi.org/10.5281/zenodo.4110309

Donepudi, P. K. (2018). Application of Artificial Intelligence in Automation Industry. Asian Journal of Applied Science and Engineering, 7(1), 7-20. http://doi.org/10.5281/zenodo.4146232

Donepudi, P. K. (2019). Automation and Machine Learning in Transforming the Financial Industry. Asian Business Review, 9(3), 129-138. https://doi.org/10.18034/abr.v9i3.494

Donepudi, P. K., Ahmed, A. A. A., Saha, S. (2020). Emerging Market Economy (EME) and Artificial Intelligence (AI): Consequences for the Future of Jobs. PalArch's Journal of Archaeology of Egypt/Egyptolog, 17(6), 5562-5574. https://archives.palarch.nl/index.php/jae/article/view/1829

Gorur, B. K., \& Calli, A. N. (2018). An Object-Oriented Agent-Based Framework for Modeling and Simulation in Aerospace. In 2018 AIAA Modeling and Simulation Technologies Conference (p. 0119). Retrieved from: https://arc.aiaa.org/doi/pdf/10.2514/6.2018-0119

Lee, S., Oh, B. S., Kim, Y., \& Park, G. (2017). High-Altitude Environment Simulation of Space Launch Vehicle in a Ground-Test Facility. Journal of the Korean Society for Aeronautical \& Space Sciences, 45(11), 914-921. Retrieved from: https://www.koreascience.or.kr/article/JAKO201734549519088.page

Lewis, B. (2016). Flight simulation in aerospace engineering education. Retrieved from: http://blewis.me/PP/resources/final-report.pdf

Linger, R. C. (2018). Behavior Computation of Aerospace Software for Validation of Security and Functionality. In 2018 AIAA SPACE and Astronautics Forum and Exposition (p. 5274). Retrieved from: https://arc.aiaa.org/doi/pdf/10.2514/6.2018-5274

O'Regan, G. (2016). Introduction to the history of computing: a computing history primer. Springer. Retrieved

from:

https://books.google.com/books?hl=en\&lr=\&id=6u16DAAAQBAJ\&oi=fnd\&pg=PR5\&dq=histo ry+of+aerospace+computing\&ots=jiODaba9EI\&sig=r99uwNkxyAzyFZPX2kbYClHV4PU

Sucharov, M. (2018). Scholarly and public engagement in Jewish politics: A typical journey liminal space in ten short chapters. Shofar: An Interdisciplinary Journal of Jewish Studies, 36(3), 143-163. Retrieved from: https://muse.jhu.edu/article/708932/summary

$--0-$

\section{How to Cite:}

Rahman, M., \& Amin, R. (2020). Artificial Intelligence Waves on Space Computation Management: A Review Report. Engineering International, 8(2), 53-60. https://doi.org/10.18034/ei.v8i2.500 